\title{
Oncological validation of bone turnover markers c-terminal telopeptide of type I collagen (1CTP) and peptides n-terminal propeptide of type I procollagen (P1NP) in patients with prostate cancer and bone metastases
}

\author{
Stefan Aufderklamm ${ }^{1}$, Jörg Hennenlotter ${ }^{1}$, Steffen Rausch ${ }^{1}$, Cornelia Bock ${ }^{1}$, Eva Erne ${ }^{1}$, \\ Christian Schwentner ${ }^{2}$, Arnulf Stenzl ${ }^{1}$ \\ ${ }^{1}$ Department of Urology, Eberhard-Karls University Tübingen, Tübingen, Germany; ${ }^{2}$ Department of Urology, Diakonieklinikum Stuttgart, \\ Stuttgart, Germany \\ Contributions: (I) Conception and design: S Aufderklamm, J Hennenlotter, C Schwentner, A Stenzl; (II) Administrative support: S Aufderklamm, E \\ Erne, S Rausch; (III) Provision of study materials or patients: S Aufderklamm, A Stenzl; (IV) Collection and assembly of data: S Aufderklamm; (V) \\ Data analysis and interpretation: S Aufderklamm, J Hennenlotter, C Bock; (VI) Manuscript writing: All authors; (VII) Final approval of manuscript: \\ All authors. \\ Correspondence to: Stefan Aufderklamm, MD. Department of Urology, Eberhard-Karls-University Tübingen, Hoppe-Seylerstrasse 3, \\ 72076-Tübingen, Germany. Email: stefan.aufderklamm@med.uni-tuebingen.de.
}

Background: Bone formation markers c-terminal telopeptide of type I collagen (1CTP) and peptides n-terminal propeptide of type I procollagen (P1NP) were reported to be increased in patients with prostate cancer (PC) and bone metastases. The objective of the presented study was to investigate the utility of serum 1CTP and P1NP values in the diagnosis of bone metastases and in predicting oncological outcome in patients with PC.

Methods: In total, serum samples of 186 patients were included retrospectively including 53 (28.50\%) benign prostatic hyperplasia (BPH) patients and 133 (71.50\%) PC-patients. The group of patients with PC consisted of 58 patients with non-metastatic PC (cM0) (43.61\%) and 70 (52.63\%) patients with bone metastases (cM1b). Serum 1CTP and P1NP were measured by radioimmunoassay (RIA). Results were compared to clinical variables including oncologic follow-up data by univariate and multivariate analyses.

Results: Median 1CTP concentrations were significantly higher in patients with PC compared to the BPH group [5.08 (range, 1.73-158.00) vs. 4.00 (range, 2.18-34.19) $\mu \mathrm{g} / \mathrm{L}, \mathrm{P}=0.019]$. However, no significant difference of P1NP levels could be shown for these groups. With median values of $6.04(1.73-158.00)$ and $3.91 \mu \mathrm{g} / \mathrm{L}(2.04-34.51)$ for 1CTP and 48.60 (9.12-1,074.37) and 33.90 (8.72-149.30) for P1NP both markers were altered in cM1b patients compared to $\mathrm{cM} 0$ patients $(\mathrm{P}=0.001$ each). Furthermore, cancer-specific survival (CSS) and overall survival (OS) were significantly shorter in cM1b patients with higher 1CTP concentrations $(\mathrm{P}=0.037$ and $\mathrm{P}=0.019$, respectively), whereas no associations of $\mathrm{P} 1 \mathrm{NP}$ and outcomes were observed.

Conclusions: The present study confirms that increased levels of 1CTP and P1NP concentrations are associated with presence of metastatic disease in the bone. Moreover, these markers are able to predict clinical course in PC patients with bone metastases. The potential use of these markers for treatment selection in advanced PC remains to be determined.

Keywords: Bone metastases; bone turnover markers; c-terminal telopeptide of type I collagen (1CTP); peptides n-terminal propeptide of type I procollagen (P1NP); prostate cancer (PC)

Submitted Jul 27, 2020. Accepted for publication Nov 09, 2020.

doi: $10.21037 /$ tau-20-1120

View this article at: http://dx.doi.org/10.21037/tau-20-1120 


\section{Introduction}

Early detection of bone metastases in PC can help to avoid severe complications and influence patient's quality of life $(1,2)$. The two marker peptides n-terminal propeptide of type I procollagen (P1NP) and c-terminal telopeptide of type I collagen (1CTP) derive from bone metabolism and are increased in case of elevated bone turnover due to metastatic bone disease (3-5). Hence, measurement of 1CTP and P1NP may help to identify patients with bone metastases. Furthermore, a potential correlation of these markers with survival, disease progression and skeletalrelated events (SRE) has been shown $(6,7)$. P1NP has been successfully investigated as an indicator for the presence of a carcinoma of the prostate with osseous metastases $(3,8-10)$. Moreover, the metabolic product of type I collagen 1CTP was shown to be helpful in the early detection of bone lesions in prostate, lung and breast cancer and has been shown to be associated with outcomes $(3,5,11,12)$.

Although the results of previous studies are promising P1NP and 1CTP are not considered as standard markers in clinical routine so far. The objective of the presented study was to validate the utility of the bone turnover markers P1NP and 1CTP in differentiating patients with and without bone metastases from prostate cancer (PC). Furthermore, we aimed to assess potential associations between levels of these bone turnover markers and outcome of patients with advanced PC. We present the following article in accordance with the REMARK reporting checklist (available at http://dx.doi.org/10.21037/tau-20-1120).

\section{Methods}

The study was conducted in accordance with the Declaration of Helsinki (as revised in 2013). The informed consent was obtained from all patients. The study was approved by the institutional review board of the EberhardKarls-University Tübingen, Germany (643/2015 BO1). A total number of 186 patients with histologically proven benign prostatic hyperplasia (BPH) (histology by transurethral prostate resection or prostate biopsy) or PC were included retrospectively in this study. Between 2002 and 2007, the bone metabolism markers P1NP and 1CTP were determined during the regular clinical examination at the Department of Urology, Tübingen. Patients with PC were subdivided in patients with verified PC without evidence of bone metastases (PCcM0) and patients with radiologically proven bone metastases (PCcM1b).
Five patients could not be assigned to one of these groups at the time of blood taking. In addition to the age and prostate-specific antigen (PSA)-value, other parameters such as primary tumor treatment, Gleason score, T- and M-stage, the medical treatment at the time of blood collection and the death rate were determined. The medical treatment at the time of blood collection includes both the androgen deprivation therapy (ADT) (nonsteroidal antiandrogens and gonadotropin-releasing hormone agonists) and the therapy with bisphosphonates. The follow-up regarding cancerspecific survival (CSS) and overall survival (OS) was carried out by reviewing the database of the University Hospital Tübingen, the Cancer Comprehensive Center Tübingen and a questionnaire for patient's general practitioners and urologists.

The patient characteristics of the different groups of patients with $\mathrm{BPH}, \mathrm{PC}$ total, $\mathrm{PCcM} 0$ and $\mathrm{PCcM} 1 \mathrm{~b}$ are listed in Table 1.

The determination of 1CTP and P1NP was carried out by competitive radioimmunoassay (125I RIA kits UniQ, \#67034 for P1NP and \#68601 for 1CTP, Matritech, Freiburg, Germany) according to the user instructions provided by Orion Diagnostica Oy, Espoo (Finland). A calibration curve with the aid of semi-logarithmic millimeter paper was used as method of calculation of the respective peptide concentrations.

Results were compared to clinical and histopathological data by Wilkoxon/Kruskal-Wallis-tests. To evaluate CSS and OS Kaplan Meier estimates and log-rank tests were used. Univariate and multivariate Cox regression analyses were performed including 1CTP, PSA doubling time (length of time in months for the PSA level to double), age and type of metastases (M1b only versus additional M1a or M1c) and compared to CSS and OS. JMP $7.0^{\circledR}$ software was used and $P$ values $\mathrm{P}<0.05$ were considered significant.

\section{Results}

In 133 patients (71.50\%), PC was histologically diagnosed, whereas $53(28.50 \%)$ patients were included with histologically proven $\mathrm{BPH}$. The PC subgroups contained 58 (43.61\%) PCcM0 and 70 (52.63\%) PCcM1b patients. In $5(3.76 \%)$ patients the $M$-status was unknown at time of blood sampling. The mean age of the total cohort was 66.70 (range, 43-86) years, 66.25 (range, 43-81) years in PC patients and 67.79 (range, 49-80) years in patients with BPH.

Eighteen $(25.71 \%)$ out of 70 patients who had bone 
Table 1 Patient characteristics of the total collective ( $\mathrm{n}=186)$ subdivided into the following subgroups: BPH, PC total, PCcM0 and PCcM1b

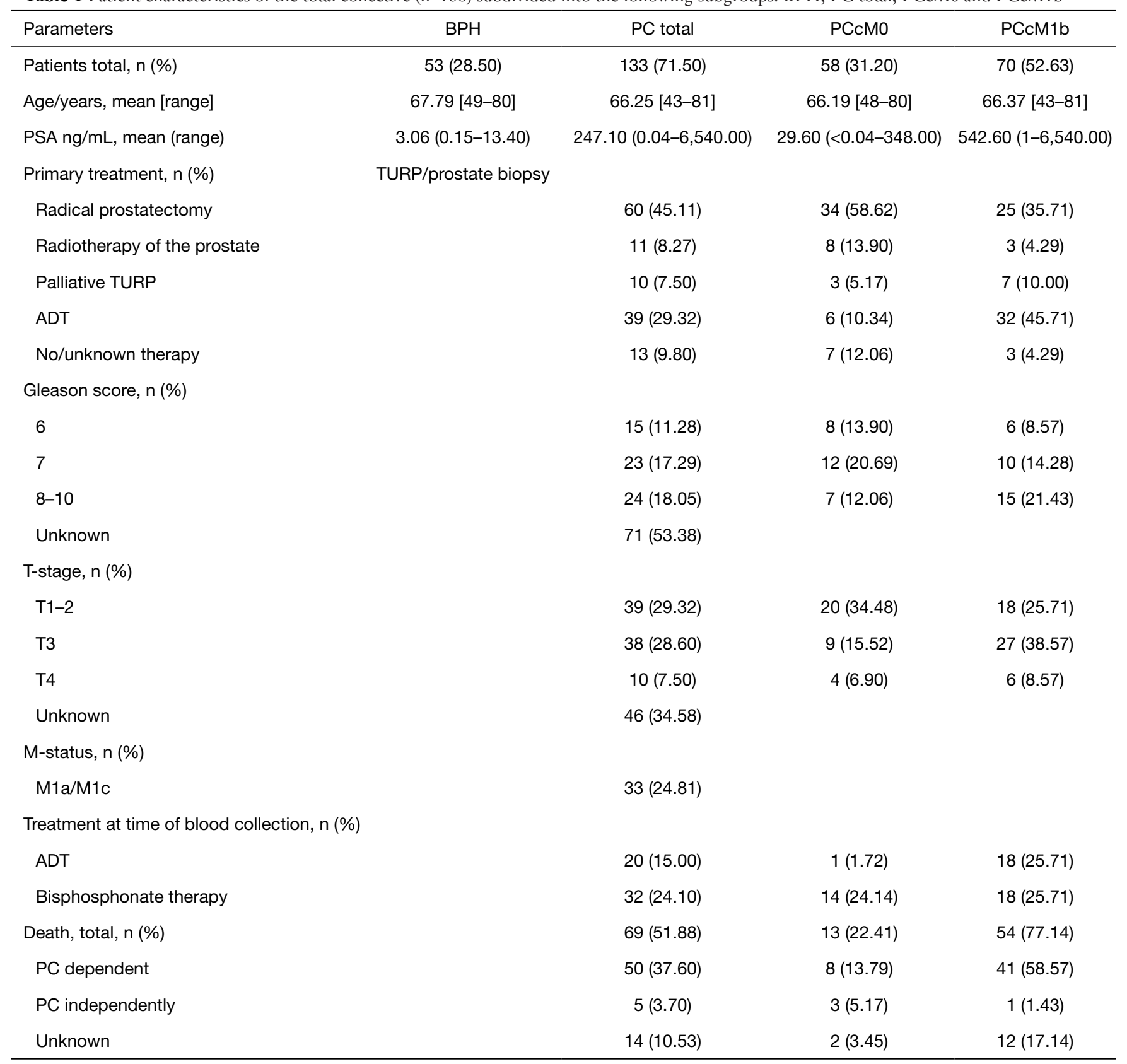

$\mathrm{BPH}$ : benign prostatic hyperplasia; PC total: patients with prostate cancer; PCcM0: patients with PC without bone metastases; PCcM1b: patients with PC and bone metastases. PSA, prostate-specific antigen; TURP, transurethral resection of the prostate; ADT, androgen deprivation therapy; M1a, extraregional metastases; M1c, visceral metastases.

metastases at the time of $1 \mathrm{CTP}$ and $\mathrm{P} 1 \mathrm{NP}$ examination were already receiving bisphosphonate therapy. Likewise, $18(25.71 \%)$ patients had an antihormonal treatment at the time of blood collection. In 33 (24.81\%) of all PC patients, additional extraregional lymph node metastases (M1a, 28 patients, $21.1 \%$ ) or visceral metastases (M1c, 5 patients,
$3.8 \%)$ were seen during the disease course.

The median follow-up period was 60 (range, 6-186) months in PCcM0 patients and 40 (range, $1-142$ ) months in PCcM1b patients. Follow-up could not be assessed in 15 patients (11.28\%).

The different serum concentrations of 1CTP und P1NP 
Table 2 Serum concentrations (median and range) of 1CTP und P1NP in the different groups: BPH, PC total, PCcM0, PCcM1b

\begin{tabular}{lcccc}
\hline Parameters & BPH & PC total & PCcM0 & PCcM1b \\
\hline ICTP $\mu g / L(R I A)$, median (range) & $4.00(2.18-34.19)$ & $5.08(1.73-158.00)$ & $3.91(2.04-34.51)$ & $6.04(1.73-158.00)$ \\
PINP $\mu g / L(R I A)$, median (range) & $38.25(11.70-247.52)$ & $41.49(8.72-1,074.37)$ & $33.90(8.72-149.30)$ & $48.60(9.12-1,074.37)$ \\
\hline
\end{tabular}

$\mathrm{BPH}$ : benign prostatic hyperplasia; PC total: patients with prostate cancer; PCcM0: patients with PC without bone metastases; PCcM1b: patients with PC and bone metastases. 1CTP, c-terminal telopeptide of type I collagen; P1NP, peptides n-terminal propeptide of type I procollagen; RIA, radioimmunoassay.
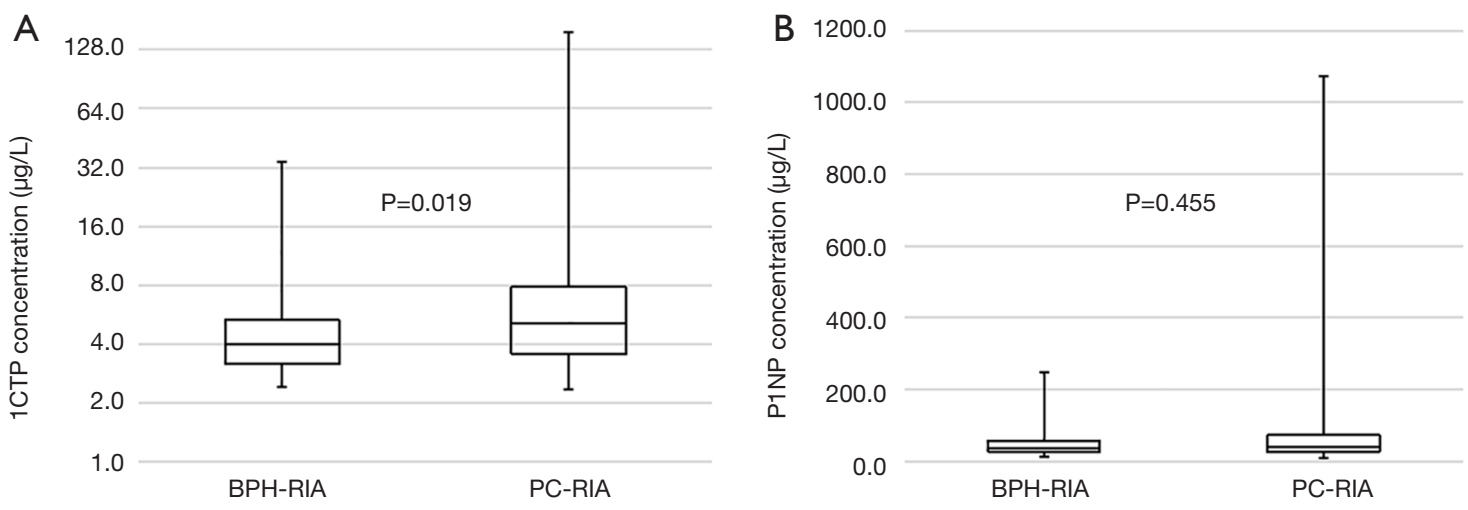

Figure 1 Differences in median 1CTP (A) and P1NP (B) concentrations in all patients with BPH and PC. 1CTP, c-terminal telopeptide of type I collagen; P1NP, peptides n-terminal propeptide of type I procollagen; BPH, benign prostatic hyperplasia; PC, prostate cancer; RIA, radioimmunoassay.

are listed in Table 2.

\section{CTP and P1NP}

Median serum concentrations of 1CTP were significantly higher in $\mathrm{PC}$ compared to patients with $\mathrm{BPH}$ [5.08 (range, 1.73-158.00) vs. 4.00 (range, 2.18-34.19) $\mu \mathrm{g} / \mathrm{L}$ respectively, $\mathrm{P}=0.019$ (Figure $1 A$ ). The measured median serum concentration of P1NP was 41.49 (range, 8.72-1,074.37) in PC total and 38.25 (range, 11.7-247.52) $\mu \mathrm{g} / \mathrm{L}$ in patients with $\mathrm{BPH}$ without significant differences, $\mathrm{P}=0.455$ (Figure $1 B$ ).

In patients with $\mathrm{PC}$, the median serum concentration of 1CTP was significantly higher in PCcM1b patients compared to the PCcM0 group [6.04 (range, 1.73-158.00) vs. 3.91 (range, 2.04-34.51) $\mu \mathrm{g} / \mathrm{L}, \mathrm{P}=0.001$ (Figure $2 A$ ). The median value of the P1NP determination was significantly higher in the PCcM1b group compared to the PCcM0 group and was 48.60 (range, 9.12-1,074.37) vs. 33.90 (range, 8.72-149.30) $\mu \mathrm{g} / \mathrm{L}, \mathrm{P}=0.001$ (Figure 2B).

In PCcM1b patients, CSS was significantly shorter in the group with 1CTP concentrations above the median [median serum concentration of $6.04 \mu \mathrm{g} / \mathrm{L}, \mathrm{P}=0.037$ (Figure $3 A$ ).
The survival rates for 2 and 5 years were $50 \%$ and $33 \%$ in patients with 1CTP concentration above the median and $75 \%$ and $52 \%$ in patients with 1CTP concentrations below the median respectively.

OS was significantly shorter in PCcM1b and $1 \mathrm{CTP} \geq$ the median of $6.04 \mu \mathrm{g} / \mathrm{L}$ compared to patients with 1CTP $<$ the median, $\mathrm{P}=0.019$. With regard to CSS and OS, neither a significant correlation in patients with $\mathrm{P} 1 \mathrm{NP}$ $\geq$ the median $(\mathrm{P}=0.214$ and $\mathrm{P}=0.281$, respectively), nor a higher risk of cancer-specific mortality and overall mortality could be verified ( $\mathrm{HR}=1.52,95 \% \mathrm{CI}, 0.78-3.02 ; \mathrm{P}=0.214$ and $\mathrm{HR}=1.52,95 \% \mathrm{CI}, 0.86-2.72 ; \mathrm{P}=0.147$, respectively), (Figure $3 B$ and Figure 4A,B).

The average survival rates for 2 and 5 years revealed $47 \%$ and $27 \%$ in patients with $1 \mathrm{CTP} \geq$ the median and $72 \%$ and $54 \%$ in patients with 1CTP less than the median.

Patients with higher 1CTP concentrations showed a significantly elevated risk for cancer-specific mortality compared to the group with 1CTP concentration below the median, $\mathrm{HR}=2.15$ (95\% CI, 1.06-4.81), $\mathrm{P}=0.032$. Moreover, the risk for overall mortality was higher in patients with elevated 1CTP serum concentrations, HR 

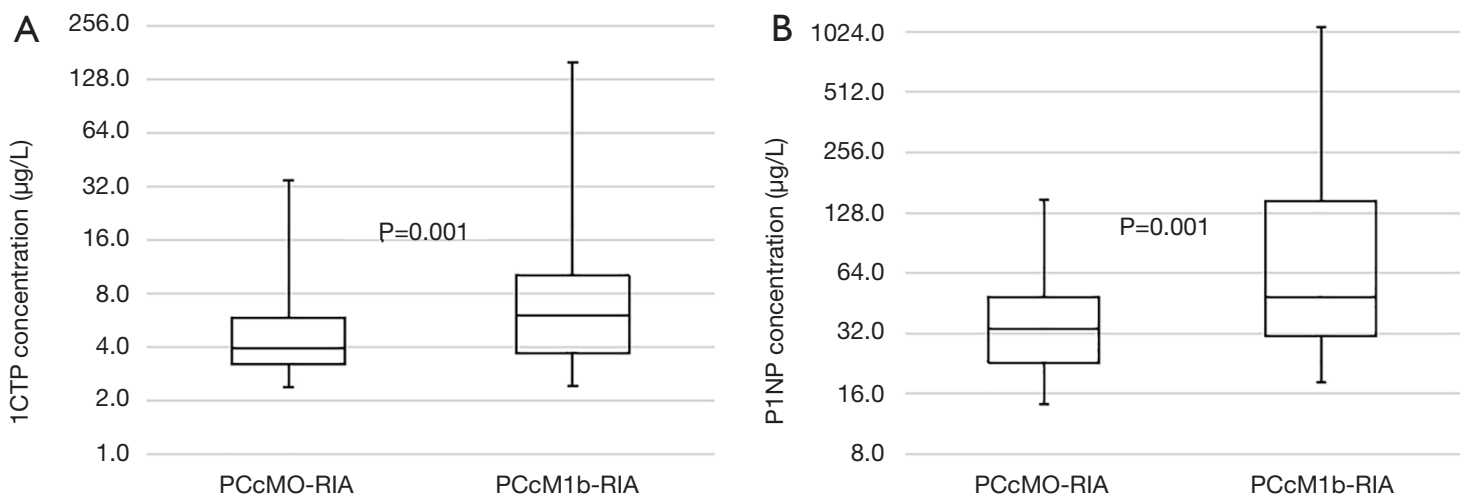

Figure 2 Median serum concentrations of 1CTP (A) and P1NP (B) in patients with (M1b) and without bone metastases (M0). 1CTP, c-terminal telopeptide of type I collagen; P1NP, peptides n-terminal propeptide of type I procollagen; RIA, radioimmunoassay.

A

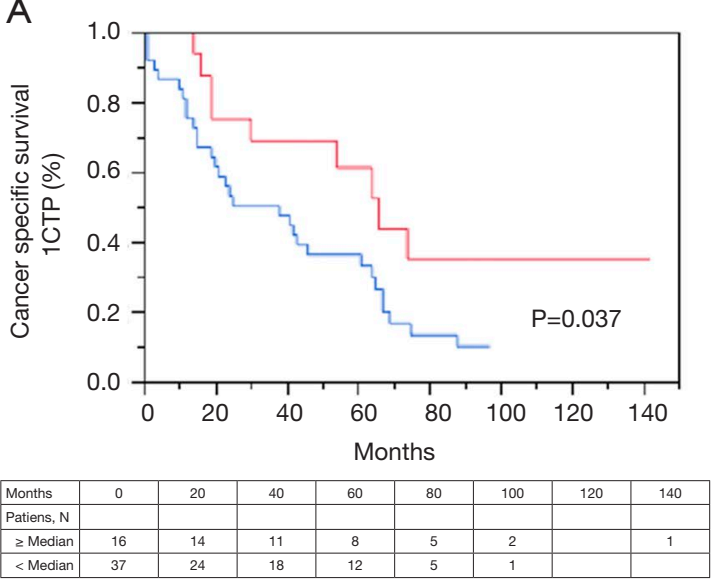

B

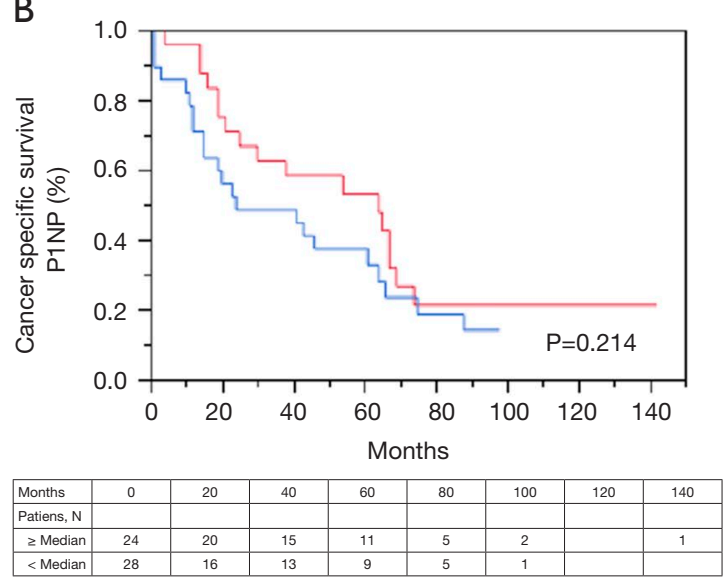

Figure 3 Kaplan-Meier curves for CSS in patients with bone metastases and 1CTP (A) and P1NP (B) measurement. Blue curves: marker concentration $\geq$ median, red curves: marker concentration < median value. CSS, cancer-specific survival; 1CTP, c-terminal telopeptide of type I collagen; P1NP, peptides n-terminal propeptide of type I procollagen.

$=2.15$ (95\% CI, 1.14-4.41), $\mathrm{P}=0.0161$. Multivariate analysis showed only 'additional M1a/M1c' to be an independent risk factor for OS $(\mathrm{P}=0.015)$, Table 3.

\section{Discussion}

Clinical data about the use of bone markers are limited (7). Although the bone formation markers 1CTP and P1NP are already used in oncology, they are not considered as standard markers in clinical routine so far $(3,9)$. The aim of the presented study was to investigate levels of bone turnover markers P1NP and 1CTP in patients with PC and benign controls and correlate levels with clinical stage and outcome. The measured median serum concentrations of 1CTP were significantly higher in PC compared to patients with $\mathrm{BPH}(\mathrm{P}=0.0195)$. In accordance with these findings, Yoshida et al. have also demonstrated that the measured serum concentration for 1CTP was significantly higher in the group of patients with PC regardless of their status of bone metastasis compared to the group of patients with BPH (13). Another study group showed higher 1CTP concentrations in $\mathrm{PC}$ patients with and without bone lesions compared to BPH patients (14). The fact that bone formation markers are higher in patients with PC can be explained by tumor cell induced "osteomimicry" which can alter the physiology of the osteoblasts. This results 

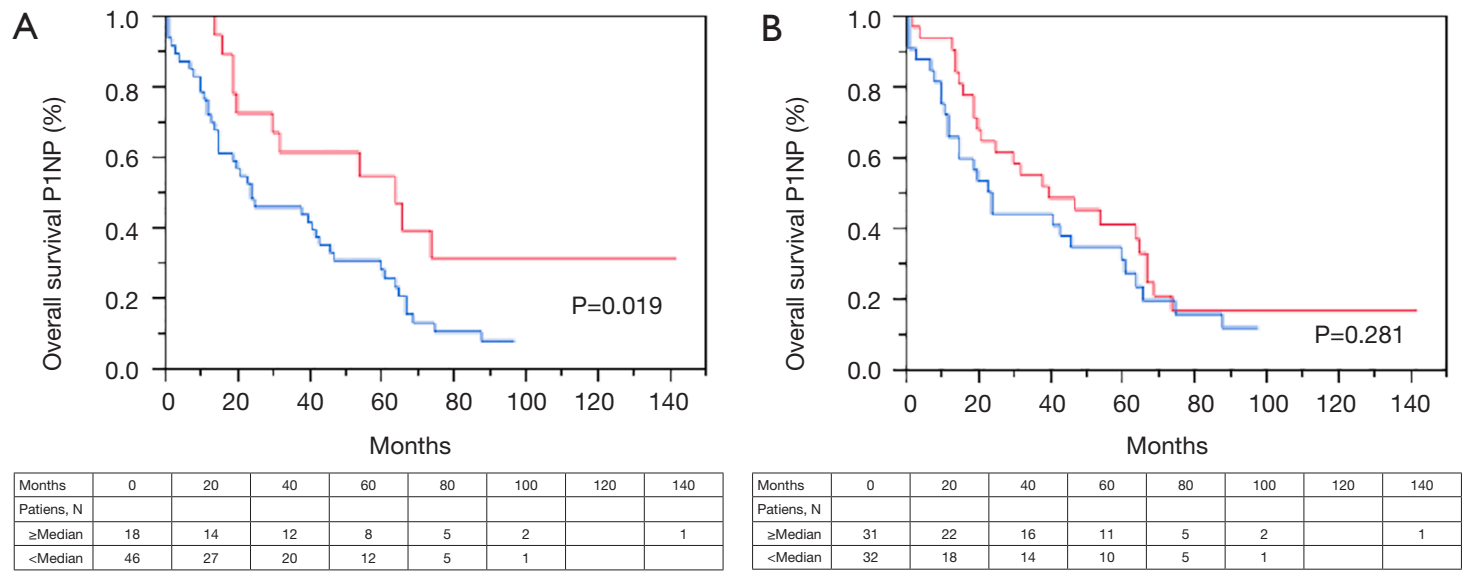

Figure 4 Kaplan-Meier curve for OS in patients with bone metastases and 1CTP (A) and P1NP (B) measurement. Blue curves: marker concentration $\geq$ median, red curves: marker concentration < median value. OS, overall survival; 1CTP, c-terminal telopeptide of type I collagen; P1NP, peptides n-terminal propeptide of type I procollagen.

Table 3 Cox regression analyses for CSS and OS in patients with prostate cancer

\begin{tabular}{|c|c|c|c|c|c|c|}
\hline Parameters & \multicolumn{3}{|c|}{ CSS } & \multicolumn{3}{|c|}{ os } \\
\hline 1CTP-RIA $\geq$ median vs. < median & 1.69 & $0.65-5.24$ & 0.296 & 1.52 & $0.63-4.25$ & 0.362 \\
\hline PSA-DT $\geq 6$ vs. $<6$ months & 1.38 & $0.53-3.66$ & 0.503 & 1.36 & $0.58-3.21$ & 0.475 \\
\hline$\geq$ Age median vs. < age median in PCcM1b & 1.80 & $0.71-4.55$ & 0.214 & 1.41 & $0.62-3.18$ & 0.400 \\
\hline
\end{tabular}

CSS, cancer-specific survival; OS, overall survival; PSA, prostate-specific antigen; DT, doubling time; M1b, bone metastases; M1a, extraregional lymph node metastases; M1c, visceral metastases.

in the expression of bone markers, matrix proteins and paracrine factors (15). Other data show, that P1NP could also be applied in the early detection of bone metastases. In contrast to previously published data with on P1NP and $\mathrm{PC}$ we could not detect any significant differences between patients with PC and BPH (16). However, the median concentration for P1NP in the investigated group was significantly below that of the other studies. This can be explained by a possible different metastasis load and thus a missing significant difference to patients with $\mathrm{BPH}$ (16).

With regards to P1NP and 1CTP as markers for bone metastases, we could confirm that both markers were able to distinguish between PC-patients with and without bone metastases. These data are congruent to other authors' results. Several authors showed that patients with PC and bone metastases had a significantly higher P1NP levels than those without bone metastases $(10,17)$. Furthermore, serum concentrations of P1NP and 1CTP correlated significantly with metastases in bone scans (13). In comparison to imaging methods such as CT or bone scans, these two serum markers are easier to access, less expensive and without any side effects such as radiation exposure or contrast media induced renal insufficiency. However, a disadvantage compared to imaging is the lack of correlation with lymph nodes or visceral metastases. During the disease course, a downward trend of these markers has been shown when ADT was used in PC patients and bone metastases (13). This allows an additional monitoring, especially when P1NP and 1CTP are used serially in PC patients and bone metastases (13). Another advantage of this marker for osteoblastic bone activity is that it is apparently independent of the local tumor occurrence and visceral metastases (16). Furthermore, P1NP could also be applied in the early detection of bone metastases. In this context, a study published 
by Koopmans et al. verified that increased $\mathrm{P} 1 \mathrm{NP}$ values may occur in patients with bone metastases up to 8 months before lesions can be detected by bone scintigraphy (3). In addition to P1NP we could also show the diagnostic potential of 1CTP for bone metastases in patients with PC. This seems to correspond with the data of other authors. Koopmans et al. showed that 1CTP allows discrimination between patients with $\mathrm{PC}$ and bone metastases and those without metastases or without PC, respectively. In their patient population ADT did not seem to influence levels of 1CTP (3). Additionally, in another study 1CTP turned out to be more reliable as a predictor for the presence of osseous lesions compared to PSA, the total alkaline phosphatase (AP) and bone-specific AP (18). These data underline the thesis that an increased amount of bone metabolites seems to occur in the serum in case of osseous lesions. The concentrations we measured for 1CTP and P1NP allow for a diagnostic prediction of bone metastases in patients with PC. Hence, discrimination between patients with and without bone metastases seems to be possible. Whether our median serum concentrations can be used as generally valid cut-off values for the presence of bone metastases has to be investigated in further studies. Based on these findings, these two markers could also be used to monitor medical tumor therapy. Due to the retrospective design without repetitive serum marker determinations and the small number of patients receiving medical tumor therapy no correlation between the therapeutic response and marker concentration was possible. However, this could be investigated in further prospective studies.

In order to investigate a possible correlation of 1CTP and CSS, Kylmälä et al. already examined 1CTP and AP in patients with $\mathrm{PC}$ and bone metastases. Apart from the diagnostic relevance of 1CTP and AP they showed that 1CTP was able to predict the prognosis with regard to CSS (12). Another retrospective study investigated PSA and bonespecific AP in addition to 1CTP. It turned out that 1CTP was the only marker that can predict CSS (18). In our patient population time to cancer-specific death in patients with bone metastases was also associated with higher 1CTP concentrations, $\mathrm{P}=0.0372$. Given this outcome, increased 1CTP serum concentrations definitely imply a worse prognosis for these patients. The investigation of P1NP did not show a correlation with CSS in our patient population. This result partially contradicts the previous literature (17). The results of our study did not allow an exact explanation why P1NP did not correlate with CSS.
However, P1NP serum concentrations where low compared to other studies (16). A further cause might be the fact that ADT and zoledronic acid had already started at the time of blood taking influencing P1NP serum concentrations and outcome $(19,20)$. Anyhow, other authors could not show differences of bone markers between patients with and without antihormonal treatment (17).

In respect of OS we were able to show a significant correlation with increased serum concentrations for 1CTP, $\mathrm{P}=0.0199$. With regard to OS $1 \mathrm{CTP}$ and $\mathrm{P} 1 \mathrm{NP}$ were already investigated prospectively in patients undergoing bisphosphonate therapy. In the respective study, 34 of 52 patients died and these patients had significantly higher 1CTP and P1NP serum concentrations compared to the surviving patients. Further, 1CTP and P1NP exhibited a higher predictive value for mortality and development of SRE (21). In conclusion, however, 1CTP seems to be the more reliable marker.

\section{Limitations}

Limitations of the study are its retrospective nature, the low number of patients and the heterogeneous patient population with various forms of tumor therapy. Another important aspect that must be taken into consideration is the fact that $1 \mathrm{CTP}$ and P1NP vary daily in their physiological concentration. Furthermore, a renal or hepatic insufficiency may lead to an elevation of the measured serum-concentration (22). Another limitation is the fact that a few patients were already undergoing AHT or therapy with zoledronic acid at the time of 1CTP and P1NP measurement with a possible influence of the investigated parameters (i.e., PSA doubling time) (20).

\section{Conclusions}

Measurement of serum 1CTP and P1NP concentrations seems to be helpful in detecting bone metastases and may help to predict the clinical course in PC patients with bone metastases. Hence, both markers could be used for stratifying patient risk with advanced $\mathrm{PC}$ and influence treatment options with osteoprotective agents as well as other chemotherapeutic drugs for a better individualized treatment.

\section{Acknowledgments}

Funding: None. 


\section{Footnote}

Provenance and Peer Review: This article was commissioned by the Guest Editor (Tilman Todenhöfer) for the series "Management of Advanced Genitourinary Malignancies" published in Translational Andrology and Urology. The article has undergone external peer review.

Reporting Checklist: The authors have completed the REMARK reporting checklist. Available at http://dx.doi. org/10.21037/tau-20-1120

Data Sharing Statement: Available at http://dx.doi. org/10.21037/tau-20-1120

Conflicts of Interest: All authors have completed the ICMJE uniform disclosure form (available at http://dx.doi. org/10.21037/tau-20-1120). The series "Management of Advanced Genitourinary Malignancies" was commissioned by the editorial office without any funding or sponsorship. Dr. AS reports other from Alere, other from Astellas, from Ferring, other from Ipsen, other from Janssen, other from Roche, outside the submitted work. The authors have no other conflicts of interest to declare.

Ethical Statement: The authors are accountable for all aspects of the work in ensuring that questions related to the accuracy or integrity of any part of the work are appropriately investigated and resolved. The study was conducted in accordance with the Declaration of Helsinki (as revised in 2013). The informed consent was obtained from all patients. The study was approved by the institutional review board of the Eberhard-Karls-University Tübingen, Germany (643/2015 BO1).

Open Access Statement: This is an Open Access article distributed in accordance with the Creative Commons Attribution-NonCommercial-NoDerivs 4.0 International License (CC BY-NC-ND 4.0), which permits the noncommercial replication and distribution of the article with the strict proviso that no changes or edits are made and the original work is properly cited (including links to both the formal publication through the relevant DOI and the license). See: https://creativecommons.org/licenses/by-nc-nd/4.0/.

\section{References}

1. Glass TR, Tangen CM, Crawford ED, et al. Metastatic carcinoma of the prostate: identifying prognostic groups using recursive partitioning. J Urol 2003;169:164-9.

2. Smith MR, Coleman RE, Klotz L, et al. Denosumab for the prevention of skeletal complications in metastatic castration-resistant prostate cancer: comparison of skeletalrelated events and symptomatic skeletal events. Ann Oncol 2015;26:368-74.

3. Koopmans N, de Jong IJ, Breeuwsma AJ, et al. Serum bone turnover markers (PINP and ICTP) for the early detection of bone metastases in patients with prostate cancer: a longitudinal approach. J Urol 2007;178:849-53; discussion 853; quiz 1129.

4. Polak-Jonkisz D, Zwolinska D, Bednorz R, et al. Procollagen I carboxyterminal propeptide (PICP) as a bone formation marker and carboxyterminal telopeptide of type I collagen (ICTP) as a bone degradation marker in children with chronic renal failure under conservative therapy. Med Sci Monit 2003;9:CR19-23.

5. Tähtelä R, Thölix E. Serum concentrations of type I collagen carboxyterminal telopeptide (ICTP) and type I procollagen carboxy-and aminoterminal propeptides (PICP, PINP) as markers of metastatic bone disease in breast cancer. Anticancer Res 1996;16:2289-93.

6. Thurairaja R, Iles RK, Jefferson K, et al. Serum aminoterminal propeptide of type 1 procollagen $(\mathrm{P} 1 \mathrm{NP})$ in prostate cancer: a potential predictor of bone metastases and prognosticator for disease progression and survival. Urol Int 2006; 76:67-71.

7. de la Piedra C, Alcaraz A, Bellmunt J, et al. Usefulness of bone turnover markers as predictors of mortality risk, disease progression and skeletal-related events appearance in patients with prostate cancer with bone metastases following treatment with zoledronic acid: TUGAMO study. Br J Cancer 2013;108:2565-72.

8. Orum O, Hansen M, Jensen CH, et al. Procollagen type I N-terminal propeptide (PINP) as an indicator of type I collagen metabolism: ELISA development, reference interval, and hypovitaminosis $\mathrm{D}$ induced hyperparathyroidism. Bone 1996;19:157-63.

9. Pollmann D, Siepmann S, Geppert R, et al. The aminoterminal propeptide (PINP) of type I collagen is a clinically valid indicator of bone turnover and extent of metastatic spread in osseous metastatic breast cancer. Anticancer Res 2007;27:1853-62.

10. Koizumi M, Yonese J, Fukui I, et al. The serum level of the amino-terminal propeptide of type I procollagen is a sensitive marker for prostate cancer metastasis to bone. BJU Int 2001;87:348-51. 
11. Kong QQ, Sun TW, Dou QY, et al. Beta-CTX and ICTP act as indicators of skeletal metastasis status in male patients with non-small cell lung cancer. Int J Biol Markers 2007;22:214-20.

12. Kylmälä T, Tammela TL, Risteli L, et al. Type I collagen degradation product (ICTP) gives information about the nature of bone metastases and has prognostic value in prostate cancer. Br J Cancer 1995;71:1061-4.

13. Yoshida K, Sumi S, Arai K, et al. Serum concentration of type I collagen metabolites as a quantitative marker of bone metastases in patients with prostate carcinoma. Cancer 1997;80:1760-7.

14. Hosoya Y, Arai K, Honda M, et al. Serum levels of the carboxy-terminal propeptide of type I procollagen and the pyridinoline cross-linked carboxy-terminal telopeptide of type I collagen as markers of bone metastases in patients with prostate carcinoma. Eur Urol 1997;31:220-3.

15. Rucci N, Teti A. Osteomimicry: how the seed grows in the soil. Calcif Tissue Int 2018;102:131-40.

16. Klepzig M, Jonas D, Oremek GM. Procollagen type 1 amino-terminal propeptide: a marker for bone metastases in prostate carcinoma. Anticancer Res 2009;29:671-3.

17. Jung K, Lein M, Stephan C, et al. Comparison of 10 serum bone turnover markers in prostate carcinoma patients with bone metastatic spread: diagnostic and prognostic implications. Int J Cancer 2004;111:783-91.

18. Kamiya N, Suzuki H, Yano M, et al. Implications of serum bone turnover markers in prostate cancer patients with bone metastasis. Urology 2010;75:1446-51.

19. Lein M, Miller K, Wirth M, et al. Bone turnover markers as predictive tools for skeletal complications in men with metastatic prostate cancer treated with zoledronic acid. Prostate 2009;69:624-32.

20. Liberman UA, Weiss SR, Broll J, et al. Effect of oral alendronate on bone mineral density and the incidence of fractures in postmenopausal osteoporosis. The Alendronate Phase III Osteoporosis Treatment Study Group. N Engl J Med 1995;333:1437-43.

21. Jung K, Miller K, Wirth M, et al. Bone turnover markers as predictors of mortality risk in prostate cancer patients with bone metastases following treatment with zoledronic acid. Eur Urol 2011;59:604-12.

22. Melkko J, Hellevik T, Risteli L, et al. Clearance of NH2terminal propeptides of types I and III procollagen is a physiological function of the scavenger receptor in liver endothelial cells. J Exp Med 1994;179:405-12.
Cite this article as: Aufderklamm S, Hennenlotter J, Rausch S, Bock C, Erne E, Schwentner C, Stenzl A. Oncological validation of bone turnover markers c-terminal telopeptide of type I collagen (1CTP) and peptides n-terminal propeptide of type I procollagen (P1NP) in patients with prostate cancer and bone metastases. Transl Androl Urol 2021;10(10):4000-4008. doi: $10.21037 /$ tau-20-1120 